\title{
A REPRESENTATION THEOREM FOR A CONTINUOUS LINEAR TRANSFORMATION ON A SPACE OF CONTINUOUS FUNCTIONS
}

\author{
DON H. TUCKER
}

To Professor H. J. Ettlinger on the occasion of completion of fifty years of continuous and outstanding service to his students at the University of Texas.

1. Introduction. The problem of obtaining representations of continuous linear transformations has been of interest for quite some time. (For an extensive bibliography of such theorems to 1958 see [1].) This note is for the purpose of giving one more such representation.

The general setting for the problem is as follows. Suppose $X$ and $Y$ are linear normed spaces, $C$ is the set of continuous functions from the interval $[0,1]$ into $X$ and $B$ is the space $B[X, Y]$ of bounded linear transformations from $X$ into $Y$. Given a "suitable" norm on the collection $C$ and perhaps some further restrictions on $X$ and $Y$, find a representation for all (or even some subset of) the continuous linear transformations from $C$ into $Y$. If $X=Y=$ the (real or complex) numbers and $\|f\|=\max |f(t)|$, we know already the classical result given in 1909 by F. Riesz [5] giving a representation in terms of a Stieltjes integral.

A fundamental paper on the Stieltjes integral for vector valued functions was written by M. Gowurin [2] in 1936. In that paper he defines an $\omega$-property for an operator valued function $K$ as follows: Suppose for each $t \in[0,1], K(t) \in B$, then the statement that $K$ has the $\omega$-property on $[0,1]$ means that there exists an $M>0$ such that for each subdivision $0=t_{0}<t_{1} \cdots<t_{n}=1$ and each set $\left\{x_{i}\right\}_{i=0}^{n-1}$ of points in $X,\left\|\sum_{i=0}^{n-1}\left[K\left(t_{i+1}\right)-K\left(t_{i}\right)\right] x_{i}\right\| \leqq M \cdot \max _{(i)}\left\|x_{i}\right\|$. If $K(t)$ is real and $X$ is the real number system, then this is equivalent to bounded variation. Gowurin showed that if $Y$ is complete, then in order that $\int_{0}^{1} d K(t) \cdot f(t)$ should exist for all $f \in C$ it is necessary and sufficient that $K$ satisfy the $\omega$-property and furthermore that this defined a continuous linear transformation from $C$ to $Y$ if $\|f\|_{c}$ $=\max \|f(t)\|_{x}$.

Professor T. H. Hildebrandt (private communication), has raised the question as to whether the converse is true. The main purpose of this paper is to answer his question in the negative and then answer the question, "What are the transformations from $C$ into $Y$ ?" That

\footnotetext{
Presented to the Society, January 24, 1964; received by the editors September 21, 1964.
} 
the answer is "no" follows from the following example. Let $X$ be the real number system and $Y$ be $C$. If one computes the integral for the identity map of $C$ into $C$ it is found that $K$ does not have its values in $B$ except at $t=0$ and $t=1$. It does satisfy the $\omega$-property. In general, the $\omega$-property is necessary as we shall see, but the values of $K$ must be in a "weak extension" of the space $B$.

2. Weak sequential extensions of $B$ and $Y$. We shall not require completeness of either $X$ or $Y$.

Suppose $\left\{p_{n}\right\}$ is a sequence of points in a linear normed space $S$ which converges weakly but not necessarily to a point in $S$, i.e., if $s^{*} \in S^{*}$, then $s^{*}\left(p_{n}\right)$ converges to some number. We may consider $p_{n} \in S^{* *}$ and write $p_{n}\left(s^{*}\right)$ converges. Call its limit $P\left(s^{*}\right)$. Clearly $P$ is linear and from the uniform limitedness theorem the sequence $\left\{p_{n}\right\}$ is bounded and $\left\|P\left(s^{*}\right)\right\|=\lim _{n \rightarrow \infty}\left\|p_{n}\left(s^{*}\right)\right\| \leqq\left\|s^{*}\right\|$ sup $\left\|p_{n}\right\|$ so that $P$ is bounded and $\|P\| \leqq \sup \left\|p_{n}\right\|$.

DEFINITION.

$$
\left\|\left\{p_{n}\right\}\right\|=\|P\|_{s^{* *}}=\sup _{\left\|s^{*}\right\| 1}\left\|P\left(s^{*}\right)\right\|=\sup _{\left\|s^{*}\right\| \leqq 1}\left|\lim _{n \rightarrow \infty} s^{*}\left(p_{n}\right)\right| .
$$

This defines a norm on the space $S^{+}$of equivalence classes of weakly convergent sequences in $S$ and furthermore if $p_{n}$ converges weakly to $p \in S$, then $\left\|\left\{p_{n}\right\}\right\|=\|p\|_{S}$. The following inclusions hold isometrically and isomorphically: $\bar{S} \subseteq S^{+}$and $\bar{S}^{+} \subseteq S^{* *}$ where $\bar{S}$ and $\bar{S}^{+}$denote the completions of $S$ and $S^{+}$respectively.

Consider now an element $w \in B^{+}$. We want to show that $w$ represents a bounded linear transformation $\tilde{w}$ from $X$ to $Y^{+}$. Suppose $\left\{b_{i}\right\} \in w \in B^{+}, y^{*} \in Y^{*}$ and consider $y^{*}\left(b_{i} \cdot x\right)$ for $x \in X$ and notice that $y^{*}\left(b_{i}\right) \in X^{*}$ and $\left\|y^{*}\left(b_{i}\right)\right\| \leqq\left\|y^{*}\right\|\left\|b_{i}\right\|$. Now consider $x$ as fixed and $y^{*}(\cdot) x \in B^{*}$, indeed, $\left|y^{*}(b) x\right| \leqq\left\|y^{*}\right\| \cdot\|b(x)\| \leqq\left\|y^{*}\right\|\|b\|\|x\|$ so that $\left\|y^{*}(\cdot) x\right\|<\left\|y^{*}\right\|\|x\|$, hence we see that $y^{*}\left(b_{i}\right) x$ converges. Write $b_{i}(x)=x_{i} \in Y$ and we then have that $\left\{x_{i}\right\}$ converges weakly in $Y$ and hence $\left\{x_{i}\right\}$ belongs to an element $\tilde{w}(x) \in Y^{+}$and that $\tilde{w}(x)$ may be considered an element of $Y^{* *}$. Therefore $\tilde{w}$ may be considered a linear map from $X$ to $Y^{+}$. We shall now show that it is bounded. Suppose $\|x\|_{x}=1$.

$$
\|\tilde{w}(x)\|_{Y^{+}}=\sup _{\| y^{*} \leqq 1}\left|\lim y^{*}\left(b_{i}\right) x\right| \leqq \sup _{\Downarrow b^{*} \leqq 1}\left|\lim b^{*}\left(b_{i}\right)\right|=\|w\|_{B^{+}} .
$$

Therefore $\|\tilde{w}\|_{B\left[X, Y^{+}\right]} \leqq\|w\|_{B^{+}}$.

3. Extension of a linear transformation. Suppose that each of $S$ and $Y$ is a linear normed space and $T$ is a continuous linear trans- 
formation from $S$ to $Y$. It is not difficult to see that $T$ has a norm preserving extension, $T^{* *}$ to be exact, which maps $S^{+}$into $Y^{+}$.

Denote by $C R$ the space of real-valued continuous functions on $[0,1]$ with the uniform norm and suppose $T$ is a continuous linear transformation from $C$ to $Y$. For $f \in C R$ and $x \in X, T(f(t) \cdot x)$ induces a continuous linear transformation $J$ from $C R$ to $B$ such that $J(f) \cdot x$ $=T(f \cdot x)$ and $\|J\|<\|T\|$ (see [7] for details) and $J$ has a norm preserving extension which maps $(C R)^{+}$into $B^{+}$.

F. Riesz showed that $(C R)^{+}$contains the step functions as elements by considering monotonic limits of uniformly bounded sequences of continuous functions and the linear extension of this set (see [6] for details). Thus if $f_{1}, \cdots, f_{n}$ are characteristic functions of intervals and $x_{1}, \cdots, x_{n}$ are points in $X$, then $\Im\left(f_{1}\right) \cdot x_{1}+\cdots+J\left(f_{n}\right) \cdot x_{n}$ $=T\left[f_{1} \cdot x_{1}+\cdots+f_{n} \cdot x_{n}\right]$ where we make no notational distinction now between the original $T, J$ and their extensions.

4. A representation for $T$. Denote by $G_{a b}(t)$ the unit step function on $[a, b)$ and define $K(t)=\Im\left(G_{0 t}(s)\right)$ if $0<t<1, K(0)=\Im(0)$ and $K(1)$ $=J(1)$. If $0=t_{0}<\cdots<t_{n}=1$ is a subdivision of $[0,1]$ and $x_{0}, x_{1}$, $\cdots, x_{n-1}$ are points in $X$ we have that

$$
\begin{aligned}
\left\|\sum_{i=0}^{n-1}\left[K\left(t_{i+1}\right)-K\left(t_{i}\right)\right] x_{i}\right\|_{Y^{+}} & =\left\|\sum_{i=0}^{n-1} J\left(G_{t_{i} t_{i+1}}(s)\right) \cdot x_{i}\right\|_{Y^{+}} \\
& =\left\|T\left[\sum_{i=0}^{n-1} G_{t_{i} t_{i}+1}(s) \cdot x_{i}\right]\right\|_{Y^{+}} \leqq\|T\| \max \left\|x_{i}\right\|
\end{aligned}
$$

and hence $K$ has the Gowurin $\omega$-property and $W_{0}^{1} K \leqq\|T\|$ where $W_{0}^{1} K$ denotes the glb of the numbers $M$ in the definition of the $\omega$ property.

Suppose $f \in C$, then $f$ is the uniform limit of step functions $\phi_{n}(t)$ $=\sum_{i=0}^{n-1} G_{i / n,(i+1) / n}(t) \cdot f\left(\xi_{i}\right)$ where $i / n \leqq \xi_{i} \leqq(i+1) / n$ and $T\left(\phi_{n}(t)\right)$ $=\sum_{i=0}^{n-1} J\left(G_{i / n,(i+1) / n}(t)\right) \cdot f\left(\xi_{i}\right)=\sum_{i=0}^{n-1}[K((i+1) / n)-K(i / n)] \cdot f\left(\xi_{i}\right)$. It is our claim that $T\left(\phi_{n}\right)$ converges to $T(f)$ in the norm of $Y^{+}$. To this end we shall approximate the functions $\phi_{n}$ by continuous functions $\theta_{n, m}$ such that $T\left(\theta_{n, m}\right) \rightarrow T\left(\phi_{n}\right)$ as $m \rightarrow \infty$, the convergence being in the sense of $Y^{+}$and then show that $\left\|T\left(\theta_{n, m}\right)-T(f)\right\|_{Y}$ converges to zero as $n \rightarrow \infty$, the rate of convergence being independent of $m$ and then use this to establish our claim.

Denote by $\left\{\theta_{n, m}\right\}_{m \geqq 2 n}$ a sequence of continuous functions converging to $\phi_{n}(t)$ and such that for each $m,\left\|\theta_{n, m}\right\|=\left\|\theta_{n}\right\|=\max \left\|f\left(\xi_{i}\right)\right\|$. In particular $\theta_{n, m}(t)=f\left(\xi_{0}\right)$ if $0 \leqq t \leqq i / n-1 / m, \theta_{n, m}(t)=\lambda f\left(\xi_{i}\right)$ $+(1-\lambda) f\left(\xi_{i-1}\right)$ if $1 / n-1 / m \leqq t \leqq i / n$ with $0 \leqq \lambda \leqq 1, \theta_{n, m}(t)=f\left(\xi_{n-1}\right)$ if $(n-1) / n \leqq t \leqq 1$ and finally $\theta_{n, m}(t)=f\left(\xi_{i}\right)$ if $i / n \leqq t \leqq(i+1) / n-1 / m$. 
We now consider $\left\|\theta_{n, m}-f\right\|_{C}=\max \left\|\theta_{n, m}(t)-f(t)\right\|_{X}$ where this maximum value must occur in one of the four cases in the definition of $\theta_{n, m}$. A routine examination shows that in each case the norm is dominated by $2 \sigma(2 / n)$ where $\sigma(2 / n)$ denotes the maximum oscillation of $f$ on an interval of length $2 / n$. Since $f$ is continuous this number converges to zero and hence we have that $T\left(\theta_{n, m}\right)$ converges in norm to $T(f)$ and hence weakly to $T(f)$ as $n$ increases without bound. If one considers the triangular array $\left\{\theta_{n, m}\right\}_{m \geq 2 n}, n=1,2, \cdots$, with $n$ denoting the row number, we have that $T$ of the $n$th row is an element of $T\left(\phi_{n}\right)$. Furthermore, on every "path" which runs through all the rows in some monotonic fashion, we obtain uniform convergence to $f$, that is $\left\{\theta_{n_{i}, m_{i}}\right\}$ converges uniformly to $f$ as $n_{i} \rightarrow \infty$.

Now consider $T(f)$, which is a point in $Y$, as a point in $Y^{+}$, that is as an equivalence class. We want to show that the sequence $T\left(\phi_{n}\right)$ of equivalence classes converges to the equivalence class $T(f)$ in the norm of $Y^{+}$. From the method of construction, $\left\{T\left(\theta_{n, m}\right)\right\}_{m \geq 2 n}$ is an element of the equivalence class $T\left(\phi_{n}\right)$ and will be used as a representative element of that class. For a representative element of the equivalence class $T(f)$ we shall use the sequence $\left\{T\left(f_{m}\right)\right\}$ where $f_{m} \equiv f$ for each $m$. With all this in mind we have

$$
\begin{aligned}
\left\|T\left(\phi_{n}\right)-T(f)\right\|_{Y^{+}} & =\sup _{\left\|v^{*}\right\| \leqq 1}\left|\lim _{m \rightarrow \infty} y^{*}\left[T\left(\theta_{n, m}-f_{m}\right)\right]\right| \\
& \leqq \sup _{(m)}\|T\| \cdot\left\|\theta_{n, m}-f_{m}\right\|_{C}=\sup _{(m)}\|T\| \cdot\left\|\theta_{n, m}-f\right\|_{C} \\
& \leqq\|T\| \cdot 2 \sigma(2 / n)
\end{aligned}
$$

and hence $T\left(\phi_{n}\right)$ converges to $T(f)$ in the $Y^{+}$norm. It should be observed that the particular mode of subdivision is unimportant so long as the mesh fineness converges to zero. Hence we may write $T(f)=\int_{0}^{1} d K \cdot f$ where the convergence of the integral is in the $Y^{+}$norm. We may now even say that

$$
\begin{aligned}
W_{0}^{1} K & =\|T\| \text { for }\|T(f)\|_{Y}=\|T(f)\|_{Y^{+}}=\left\|\int d K \cdot f\right\|_{Y^{+}} \\
& =\lim _{n \rightarrow \infty}\left\|\sum_{i=0}^{n-1}[K((i+1) / n)-K(i / n)] \cdot f\left(\xi_{i}\right)\right\|_{Y^{+}} \\
& \leqq \sup _{(i)} W_{0}^{1} K \cdot \max \left\|f\left(\xi_{i}\right)\right\| \leqq W_{0}^{1} K \cdot\|f\|_{C}
\end{aligned}
$$

and hence $\|T\| \leqq W_{0}^{1} K$. This together with the reverse case given earlier establishes the equality.

We may now state our main result in the form of a theorem. 
THEOREM 1. If $T$ is a bounded linear transformation from $C$ into $Y$, then there exists a function $K$ on $[0,1]$ such that $K(t) \in B^{+}$, hence in $B\left[X, Y^{+}\right]$for each $t, K$ has the Gorwurin w-property with $W_{0}^{1} K=\|T\|$ and for each $f$ in $C, T(f)=\int_{0}^{1} d K \cdot f$ where the integral converges in the $Y^{+}$ norm.

The integral considered above is of the Gowurin type [2] and if one considers the elements of the approximating sums as elements of $Y^{* *}$ which is complete the usual properties hold. See [2] and [4]. If, however, one asks for more precise information as to where say $\int_{0}^{t} d K \cdot f$ might be when $0<t<1$, difficulties arise. In the example given in the introduction, these values are in $\mathrm{Y}^{+}$and not in $Y$.

Let us consider the following situation. We have given a function $K$ having the $\omega$-property with its values in $B^{+}$. Such a $K$ will determine a $T$, continuous and linear from $C$ into $\bar{Y}^{+}$. Question: Under what further conditions does such a $K$ determine a $T$ from $C$ into $Y$ ? This question has been answered by the writer and Lynn C. Kurtz [3] for the case in which $X$ and $Y$ are complete.

\section{Concerning the uniqueness of the function $K$.}

Lemma. If $K(t) \in B\left[X, Y^{+}\right]$for each $t \in[0,1]$ and $K$ has the $\omega$ property in the sense that $\left\|\sum_{\sigma}\left\{K\left(t_{i+1}\right)-K\left(t_{i}\right)\right\} x_{i}\right\|_{Y^{+}} \leqq W \cdot \max \left\|x_{i}\right\|_{X}$ for each subdivision $\sigma$ of $[0,1]$, then for each $F \in B^{*}\left[X, Y^{+}\right] F K$ is of bounded variation on $[0,1]$ and $V_{0}^{1} F K \leqq\|F\| \cdot W_{0}^{1} K$.

Proof.

$$
\begin{aligned}
\sum_{i=0}^{n-1} \mid F K\left(t_{i+1}\right) & -F K\left(t_{i}\right)\left|=\sum_{i=0}^{n-1}\right| F\left\{K\left(t_{i+1}\right)-K\left(t_{i}\right)\right\} \mid \\
& =\sum_{i=0}^{n-1} \epsilon_{i} F\left\{K\left(t_{i+1}\right)-K\left(t_{i}\right)\right\}=\left|F \sum_{i=0}^{n-1} \epsilon_{i}\left\{K\left(t_{i+1}\right)-K\left(t_{i}\right)\right\}\right| \\
& \leqq \\
& \|F\| \cdot\left\|\sum_{i=0}^{n-1} \epsilon_{i}\left\{K\left(t_{i+1}\right)-K\left(t_{i}\right)\right\}\right\|_{B\left[X, Y^{+}\right]} \\
& \|F\| \sup _{\|x\| \leqq 1}\left\|\sum_{i=0}^{n-1} \epsilon_{i}\left\{K\left(t_{i+1}\right)-K\left(t_{i}\right)\right\} x\right\|_{Y^{+}} \\
& =\|F\| \sup _{\|x\| \leqq 1}\left\|\sum_{i=0}^{n-1}\left\{K\left(t_{i+1}\right)-K\left(t_{i}\right)\right\} x_{i}\right\|_{Y^{+}} \leqq\|F\| W_{0}^{1} K .
\end{aligned}
$$

Suppose each of $K_{1}$ and $K_{2}$ has the $\omega$-property of the lemma and generate the same $T \in B[C, Y]$, then $K=K_{1}-K_{2}$ will generate the zero transformation. There is no loss in assuming $K(0)=0$. We know 
that for each $F \in B^{*}\left[X, Y^{+}\right], \int_{0}^{1} d(F K(t)) f(t)=F \int_{0}^{1} d K(t) \cdot f(t)$ for each $f \in C R$. Since $\int_{0}^{1} d K(t) \cdot g(t)=0$ for each $g \in C$, we have that $\int_{0}^{1} d K(t)$ $\cdot f(t) \cdot x=0$ for each $f \in C R$ and each $x \in X$, hence $\int_{0}^{1} d K(t) \cdot f(t)=0$ and therefore $\int_{0}^{1} d(F K(t)) \cdot f(t)=0$ for each $f \in C R$. Furthermore, $K(0)=0$ implies $F K(0)=0$ and therefore $\int_{0}^{1} d(F K(t)) \cdot 1=0$ implies $F K(1)=0$ for each $F$ so that $K(1)=0$. Now consider $f_{\tau}(t)=t$ if $0 \leqq t \leqq \tau, f_{\tau}(t)=\tau$ if $\tau \leqq t \leqq 1$ where $0 \leqq \tau \leqq 1$. For these functions we have $0=\int_{0}^{1} d(F K(t))$ $\cdot f_{\tau}(t)=-\int_{0}^{1} F K(t) d f_{\tau}(t)=-\int_{0}^{\tau} F K(t) d t$ which implies that $F K(t)=0$ a.e. The fact that $F K$ is of bounded variation shows that the exeptional set $E(F)$ must be countable and the values of $F K$ on this set are absolutely convergent.

We shall now show that if $K=K_{1}-K_{2}$ where $K_{1}$ and $K_{2}$ generate $T_{1} \in B[C, Y]$ and $T_{2} \in B[C, Y]$ respectively, then the above conditions are sufficient to show that $K$ generates the zero transformation. Suppose $K(0)=K(1)=0$ and, for each $F \in B^{*}\left[X, Y^{+}\right], F K=0$ except on a countable set $E(F)$ and its values there are absolutely convergent, then $F K$ is of bounded variation and $\int_{0}^{1} F K(t) d f(t)=0$ for each $f \in C R$. An integration by parts shows that $F \int_{0}^{1} d K(t) \cdot f(t)=0$ for each $f \in C R$ and each $F \in B^{*}\left[X, Y^{+}\right]$. We now have that $\int_{0}^{1} d K(t) \cdot f(t)=0$ for each $f \in C R$. The fact that $K=K_{1}-K_{2}$ has the $\omega$-property implies that $T(x)=\int_{0}^{1} d K(t) \cdot x(t)$ exists for each $x \in C$ and that $\|T\| \leqq W_{0}^{1} K$. Now consider

$$
x_{n}(t)=\sum_{\nu=0}^{n}\left(\begin{array}{l}
n \\
\nu
\end{array}\right) t^{\nu}(1-t)^{n-\nu} \cdot x\left(\frac{\nu}{n}\right)
$$

which converges uniformly to $x(t)$. Since $T$ is continuous, $T\left(x_{n}\right)$ converges to $T(x)$ but

$$
T\left(x_{n}\right)=\sum_{\nu=0}^{n}\left(\begin{array}{l}
n \\
\nu
\end{array}\right)\left[\int_{0}^{1} d K(t) \cdot t^{\nu}(1-t)^{n-\nu}\right] \cdot x\left(\frac{\nu}{n}\right)=0
$$

and hence $T(x)=0$ for each $x \in C$.

We may now summarize the results in the form of a theorem.

THEOREM 2. In order that two functions $K_{1}$ and $K_{2}$ should generate the same continuous linear transformation $T \in B[C, Y]$ it is necesssary and sufficient that

(i) each of $K_{1}$ and $K_{2}$ generate such a $T$ and

(ii) there exists a point $d \in B^{+}[X, Y]$ such that $K_{1}(0)-K_{2}(0)=K_{1}(1)-K_{2}(1)=d$ and for each $F \in B^{*}\left[\begin{array}{ll}X, Y^{+}\end{array}\right]$, $F\left[K_{1}(t)-K_{2}(t)\right]=F(d)$ except possibly over a countable set $E(F)$ of points and that for the points $e \in E(F), \sum_{E(F)}\left|F\left[K_{1}(e)-K_{2}(e)\right]\right|$ converges. 
In a certain sense, this theorem is not all that one might hope to have. It would be a far more interesting result if in (i) the word each could be replaced by the word one. This could be done if one could prove the converse of the lemma. Thus far, the best result along these lines that the author has obtained is as follows.

Theorem 3. If for each $t \in[0,1], K(t) \in B\left[X, Y^{+}\right]$and $K$ is such that for each $F \in B^{*}\left[X, Y^{+}\right], F K$ is of bounded variation on $[0,1]$, then there exists a number $W>0$ such that for each partition $\sigma: 0=t_{0}$ $\leqq t_{1} \leqq \cdots \leqq t_{n}=1$ and each sequence $\left\{r_{i}\right\}, i=0, \cdots, n-1$ of real numbers, $\left\|\sum_{i=0}^{n-1}\left\{K\left(t_{i+1}\right)-K\left(t_{i}\right)\right\} r_{i}\right\| \leqq W \max \left|r_{i}\right|$.

Proof. For each $F \in B^{*}\left[X, Y^{+}\right]$and each $f \in C R, \int_{0}^{1} d(F K(t)) \cdot f(t)$ exists. Since $F \int_{0}^{1} d K(t) \cdot f(t)=\int_{0}^{1} d(F K(t)) \cdot f(t)$ we have that $\int_{0}^{1} d K(t) \cdot f(t)$ exists in $B^{+}\left[X, Y^{+}\right]$for each $f \in C R$. Denote by $X_{1}$ the real numbers and by $Y_{1}$ the space

$$
\overline{B^{+}\left[\left(X, Y^{+}\right)^{-}\right.}
$$

then $B\left[X_{1}, Y_{1}\right]=\dot{Y}_{1}$ and the space $C_{1}$ of continuous functions from $[0,1]$ into $X_{1}$ is $C R$. Furthermore, since $B\left[X, Y^{+}\right]$is a subset of $Y_{1}=B\left[X_{1}, Y_{1}\right]$ we have $K(t) \in B\left[X_{1}, Y_{1}\right]$ for each $t \in[0,1] . Y_{1}$ is complete and $\int_{0}^{1} d K(t) \cdot f(t)$ exists for each $f$ in $C_{1}$, the convergence being in the norm of $Y_{1}$. Gowurin's result then establishes the theorem.

Professor Hildebrandt (private communication) has obtained a similar result by other methods.

It would also be of some interest to know just how much (ii) implies about the function $K=K_{1}-K_{2}$. For example, one might be tempted to think that $F K(t)=0$ a.e. for each $F \in B^{*}\left[X, Y^{+}\right]$implies that $K(t)=0$ a.e. This is not true.

The following example is due to a referee.

Let $X=R$ and $Y=C_{0}$, the space of all real-valued functions $f$ on the interval $[0,1]$ such that for each $e>0$, the set of all $s$ such that $|f(s)|>e$ is finite, the space $Y$ having the sup norm. For $t \in[0,1]$, let $K(t)$ be an operator from $R$ to $C_{0}$ defined by $(K(t) r)(s)=r$ if $t=s$, and 0 if $t \neq s$, where $r \in R ; t, s \in[0,1]$. It is easily shown that $K$ has the $\omega$-property and $K(t) \neq 0$ for each $t$. Furthermore, $B[X, Y]^{*}=Y^{*}$ where $y^{*} \in Y^{*}$ is determined by $y^{*}(f)=\sum_{i=0}^{\infty} f\left(s_{i}\right) y^{*}\left(K\left(s_{i}\right)\right)$ where $s_{i}$ are the points at which $f$ is not zero. The sum being independent of order. It is then seen that $y^{*}(K(t)) \neq 0$ for at most a countable set of points $t$.

6. Weak convergence of operators. We next turn our attention to the question of weak convergence of a sequence $\left\{T_{n}\right\}$. By weak con- 
vergence we mean that for each $g \in C$ and each $y^{*} \in Y^{*}, y^{*} T_{n} g$ converges, i.e., $\left\{T_{n} g\right\}$ is a representative of some element in $Y^{+}$. We assume that $K_{n}(0)=0$ for each $n$. This can always be attained, hence there is no loss. A slight refinement of the Riesz argument [5] for the real case gives the following theorem.

TheOREM 4. Suppose $X$ is complete, then for a sequence $\left\{T_{n}\right\}$ of elements of $B[C, Y]$ where $T_{n} g=\int_{0}^{1} d K_{n}(t) \cdot g(t)$ and $K_{n}(0)=0$, the following two statements are equivalent:

(i) $\left\{T_{n}\right\}$ converges weakly.

(ii) There exists a number $M>0$ such that $\left\|T_{n}\right\| \leqq M$ for each $T_{n}$ and if $y^{*} \in Y^{*}$, and $x \in X$, then $y^{*} K_{n}(1) \cdot x$ converges as $n \rightarrow \infty$ and, for $0<\tau<1, y^{*} \int_{0}^{\tau} K_{n}(t) d t \cdot x$ converges as $n \rightarrow \infty$.

Proof. For the necessity we notice that $y^{*} T_{n}(1 \cdot x)=y^{*}\left[K_{n}(1) \cdot x\right.$ $\left.-K_{n}(0) \cdot x\right]=y^{*} K_{n}(1) \cdot x$ hence $y^{*} K_{n}(1) \cdot x$ converges. With $f_{\tau}$ as in the proof of Theorem 2, we have $y^{*} T_{n}\left(f_{\tau} \cdot x\right)=y^{*} \int_{0}^{1} d K_{n} \cdot f_{\tau} \cdot x$ $=-y^{*} \int_{0}^{\tau} K_{n}(t) \cdot d t \cdot x$ converges. That $\left\|T_{n}\right\|$ are uniformly bounded follows at once from the fact that $C$ is complete if $X$ is complete and making use of the uniform boundedness principle.

The sufficiency follows then from the fact that the functions $\sum_{i=0}^{n} f_{\tau_{i}}(t) \cdot x_{i}$ are dense in $C$ and the property is sufficient for such functions.

\section{BIBLIOGRAPHY}

1. N. Dunford and J. T. Schwartz, Linear operators, Vol. I, Interscience, New York, 1958.

2. Mark Gowurin, Über die Stieltjessche Integration abstrakter Functionen, Fund. Math. 27 (1936), 254-268.

3. Lynn C. Kurtz and D. H. Tucker, Vector-valued summability methods on a linear normed space, Proc. Amer. Math. Soc. 16 (1965), 419-428.

4. J. S. MacNerney, Stieltjes integrals in linear spaces, Ann. of Math. 61 (1955), 354-367.

5. F. Riesz, Sur les operations functionelles linéaires, C. R. Acad. Sci. 149 (1909), 974-977.

6. F. Riesz and B. Sz.-Nagy, Functional analysis, Ungar, New York, 1955.

7. D. H. Tucker, $A$ note on the Riesz representation theorem, Proc. Amer. Math. Soc. 14 (1963), 354-358.

UNIVERSITY OF UTAH 